\title{
A Review on an Efficient Technique for Detection and Classification of Early Stage Tumor
}

\author{
Sangeeta, H. Nagendra
}

\begin{abstract}
To reduce the death by tumor disease it is important for the classification and identification of the early stage tumor for diagnosis. The brain tumor is categorized into two types those are primary and secondary brain tumor. Again, primary brain tumor is categorized into two types those are malignant and benign tumor. Benign tumor is non-cancerous it does not affect other parts but malignant brain tumors are cancerous they may spread into spine of our body. This paper reviews various techniques utilized to classify the brain tumors with the help of MR images. Brain tumor classification can be divided into four phases as preprocessing, segmentation, feature reduction and extraction, classification. As segmentation is important step to classify the brain tumors. Median filter is efficient to eliminate the noise. Combination of $\mathrm{K}$ means cluster and otsu binarization is enough for segmentation. DWT (Discrete wavelet transform) and GLCM (Grey Level co-occurrence matrix) efficient for transform and spatial feature extraction. PCA (Principal component analysis) reduces the feature vector to maintain the classification accuracy of brain MRI images. For the performance of MRIs classification, the significant features have been submitted to KSVM (kernel support vector machine). The proposed system will reduce processing time and better accuracy can be achieved. The proposed method is validated on BRATS 2015 dataset.
\end{abstract}

Index Terms- K-means; DWT; GLCM; PCA; KSVM; MRI Classification.

\section{INTRODUCTION}

The formation of abnormal cells in the brain leads to brain tumor. Benign and malignant are the two main kinds of tumors. The tumor which starts in the brain itself is known as primary malignant tumor. Secondary tumors (metastasis tumors), spread from elsewhere. There are four grades of brain tumors according to the American Brain Tumor Association and World Health Organization [2]. Benign tumors, which are slow growing are Grade I and grade II, and are also known as low-grade tumors. Malignant tumors, which are rapid growing are grade III and grade IV and are called high-grade tumors. It is significant to detect brain tumor at the early stage and it is necessary to identify the tumor area and segment the tumor images.

Sangeeta, Electronics and communication engineering, PDA College of engineering, Kalaburagi, Karnataka, India

H. Nagendra, Electronics and communication engineering, PDA College of engineering, Kalaburagi, Karnataka, India
Magnetic resonance imaging (MRI) and computed tomography (CT) scans are two diagnostic modes which show the internal structure of the brain. MRI is an imaging technique which provides the information about human body. Due to high contrast of soft tissues and spatial resolution of brain, it is mandatory to diagnose the brain tumor by MRI component. It does not produce radiation and is non-invasive, which make it more proficient than other techniques [4]. It has a several benefits compared to remaining imaging techniques. Operator performance causes noise in MRI images and this noise leads to inaccuracies classification. It is not easy to detect and classify the brain tumors. Due to the misplaced edges, noise, low contrast of medical images it is hard to get information from these images [31]. It includes few processes, such as image segmentation, enhancement, feature selection and extraction, feature classification and reduction. The noise is reduced by using different filter methods. Gaussian, speckle, Salt and pepper noise are additive noises of MRI images and can be removed by Averaging filter, Adaptive filter, Un-sharp masking filter, Median filter and Gaussian filter [6]. Averaging filter gives the good result by computing MSE. Median filter removes the noise based on PSNR [17]. Significant step for the detection of tumor area is segmentation. The separation of image into distinct parts as per their similar properties can be done by using clustering. Clustering is one of the unsupervised segmentation methods. K-means and Fuzzy C-means algorithm are two mostly used clustering techniques. K-means clustering is an effective way that uses a fixed number of clusters prior to classify a set of data [24]. The feature extraction can be done by using Gray Level Co-occurrence Matrix (GLCM) and Discrete wavelet transform (DWT). Spatial feature extraction can be done by using GLCM and transform feature extraction can be done by DWT. The high dimensional space patterns can be made by statistical method known as principal component analysis (PCA). The multidimensionality and number of variables can be reduced by PCA and it enables the exploring of date in an easy way [40]. The classifier on the basis of machine learning comprising of supervised and unsupervised learning has become popular in recent years. K-NN, SVM and ANN are included in supervised learning. Self-organization feature map (SOFM) and fuzzy c-means are included in unsupervised [3]. The advantageous features of SVM are regularization, low test error rate, kernel trick and absence of local minima. 
The tumor classification and detection has been done by employing various techniques till now. But these techniques have drawbacks such as lack of accuracy, intensity inhomogeneity, noise, time complexity, computational complexity, feature selection, extraction and reduction, etc. DWT with KSVM classifier is proposed to overcome these limitations and also classification of the tumor is done with high accuracy by using this classifier, which denoising and segment the image, extract and reduce the feature, select the proper features for accurate classification of the tumor as benign and malignant tumor from MRI image.

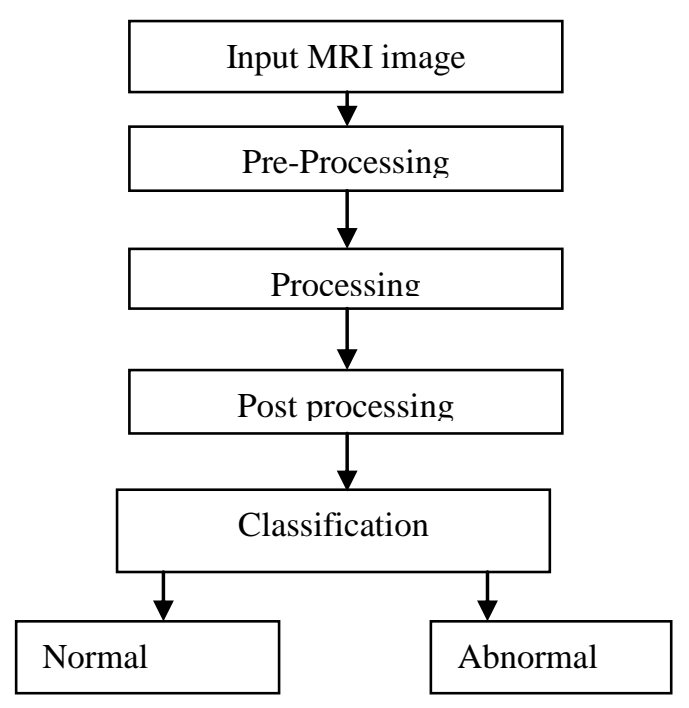

\section{Figure 1: Brain tumor classification and detection methodology}

\section{A . Pre Processing}

The detailed information of brain can be obtained by the MRI images and are considered as the input images. Input dataset is used in the form of $X=\left\{x_{1}, x_{2}, \ldots . x_{n}\right\}$. The BRATS dataset is used for the collections of images.

The first stage of this system after the preparation of the dataset is preprocessing. Some types of noise affect the magnetic resonance images which leads to degradation of the resolution [7]. The preprocessing stage enables the improvement in image quality by suppressing the noise and it also enhances the resolution of the images. De-noising and filtering at this stage is done by median filter.

\section{B. Processing}

The processing stage includes $\mathrm{K}$-means clustering and Otsu binarization. Conversion of image into binary format is done by Otsu binarization and which finds the binarization threshold automatically. It is commonly used thresholding technique. The binary image from gray-level image in image processing and in computer version can be obtained by this The reduction of structural risk can be done by original technique which also performs the clustering-based image support vector also known as binary classification method thresholding. The binary image further processed by significant clustering algorithm known as K-means. Grouping of pixels is termed as clustering. Defining the number of $\mathrm{k}$ clusters has to be done first. The cluster centers $\mathrm{k}$ is selected in random way. The calculation of distance between the pixels and the cluster centers is carried out next. Distance formula is used for the comparison of each pixel with every cluster center. The pixel is transferred to a cluster which is at a shortest distance from the pixel compared to all. This process is continuous until the clustering criterion converges.

\section{Post Processing}

Feature reduction and extraction are carried out in postprocessing. The process of feature extraction includes the extraction of significant information such as texture, color features, contrast and shape of an image. Here spatial feature extraction has been performed by GLCM and transform feature extraction is done by DWT. The relative position of pixel in an image is obtained by a statistical method called GLCM. The first texture-based technique of feature extraction was presented by R.M. Haralick. It calculates the occurrence of a pixel i having intensity $I$ in relation with other pixel $\mathrm{j}$ with distance $\mathrm{d}$ and angle $\Theta$. The element of the GLCM is formed by pixel $\mathrm{i}$ with the total number of occurrences. Resultant matrix is used to determine the features after the calculation of GLCM. The measurement of correlation, energy, contrast and homogeneity is carried out in this study. The features are extracted from the segmented image by DWT. Images are converted to frequency domain from the spatial domain. The low pass and high pass filters are used for filtering the image in both horizontal and vertical direction to perform the actual DWT. The division of image in each DWT level includes coefficients: LL, LH, HL and HH. The LL sub-bands (approximation coefficient) obtained by using the low pass and high pass filters in the horizontal and vertical directions respectively. Remaining sub-bands are called as detailed coefficients. More detailed information is extracted from the tumor using DWT. Classification complexity is increased by extra unnecessary features, require more storage memory and prolong the computational time. Thus, feature reduction is considered as a part of our proposed system. Wavelet transform dimensionality can be reduced by Principal Component Analysis (PCA) method The reduction of data dimension as per their variance and importance is done by. Emphasis of differences and similarities in standards of data and their expression is performed by PCA. Patterns can be compressed and their dimensions can be reduced, once they found without losing much of information. This reduction is useful for data representation, image compression, calculation reduction which are essential for the further processing.

\section{Classification}


presented by Vapnik. SVM is on basis of supervised techniques and is used for the problems from one-class to multiple-class classification [13]. SVM is utilized as kernel machine. The kernel enables to fit the maximum-margin hyperplane into transformed feature space, which is the significant effect of kernel trick. MRI classification is performed by KSVM. Box constraint, auto scale and kernel are the families of SVM. The selection of kernel support vector machine is on the basis of wide range of functions, such as polynomial, linear, and gaussian radial basis (GRB).

\section{LITERATURE SURVEY}

Arakeri et al., (2013) proposed an accurate and automatic computer-aided diagnosis (CAD) system on the basis of group of classifiers such as SVM, ANN and KNN to characterize the brain tumors as malignant or benign on MRI images. In pre-processing stage, the noise is suppressed by employing median filter. To segment the brain tumor on each 2D MRI image they used combination of modified FCM clustering and wavelet decomposition technique. The feature selection and ranking steps are involved in the techniques of feature selection. The feature ranking using information gain method, which selects the subset of features based on the information contribution associated with the class variable. Feature selection is done by using ICA (independent component analysis). Classification is done by using 3 classifiers such as SVM, ANN and KNN. The proposed CAD system reduces the computational cost and operational complexity as it performs the analysis of brain tumor in $2 \mathrm{D}$ features, which eliminates processing of entire 3D tumor volume. They collected 550 patients' MRI images from shirdi sai cancer hospital manipal, India. By using these 3 classifiers they achieved better accuracy such as SVM (96.42\%), ANN (94.18\%) and KNN (89.09\%).

Amien et al., (2013) instead of CAD, they used MRI images due to time consumption. They proposed the classification of brain images by employing Back-Propagation Neural Network (BPNN) which is on the basis of Pearson correlation coefficient. The proposed system consisting of 3 stages. Enhancement in the contrast and suppression of noise are done in preprocessing. Secondly, the classification of the brain tumor is performed by extracting the texture features and reduction of dimensionality are performed by using PCA and BPNN. They 58 MRI images of 3 MRI human brain dataset and they achieved $96.8 \%$ of accuracy.

Jayachandran A. et al., (2013) proposed a technique which consists of four stages. The reduction of noise is done by using anisotropic filter in the first stage. They obtained the texture features related to MRI images in the second stage. The feature reduction is done by utilizing principles component analysis in the third stage. The supervisor classifier based FSVM is utilized to classify tumor as normal and abnormal brain MR images at the last stage. They collected $80 \mathrm{MRI}$ images of non-tumor and tumor from south Indian area severity and analysis includes the processing the images and they achieved $95.80 \%$ of Classification accuracy.

Kharmega Sundararaj et al., (2014) presented the automatic brain tumor classification in CT images. The four stages of this method are preprocessing, extraction of features, feature classification and reduction. Gaussian filter is employed to reduce noise and to ensure the compatibility of image for features extraction. Extraction of intensity and texture based features are done in the second stage to classify. More accurate and efficient classification is achieved with the help of PCA by reducing the feature space dimensionality in the next stage. Experimental images are classified into abnormal and normal with the help of two classifiers during the stage of classification. The first and second classifiers are on the basis of k-nearest neighbour and Linear SVM respectively. They achieved $94 \%$ of accuracy in Linear SVM and $92 \%$ accuracy in k-NN by collecting 50 CT brain images from Department of Radiology, Rajah Muthiah Medical College Hospital (RMMCH). There are 10 normal and 40 abnormal brain images out of $50 \mathrm{CT}$ brain images.

Natteshan et al., (2015) proposed a technique known as Computer Aided Diagnosis (CAD) to classify the brain MRI automatically into non tumor and tumor. Contrast Limited Adaptive Histogram Equalization (CLAHE) and wiener filter are used to preprocess the input images. The tumor region is then extracted using the intensity metric. Support Vector Machine (SVM) and Neural network classifiers are used to classify the brain tumor. They collected 9 non tumor and 83 tumor affected grayscale images from DICOM. They achieved accuracy of $85.40 \%$ with the help of SVM classifier provided quadratic kernel function, which performs more efficient than Radial Basis Function (RBF) kernel function $(83.61 \%)$

Anitha. V et al., (2016) introduced an approach called two-tier classification for classification methodology and the segmentation is performed by employing adaptive pillar K-means algorithm. Discrete wavelet, transform blend wavelets are used to extract the feature from neural network trains and K-nearest neighbor are used for consequent train of the resultant filter factors. The preferable performance i.e., the classification of brain tumors in double training process is done two-tier system compared to traditional method of classification. It is implemented in MATLAB R2013a. The patterns of the same classes having small intra-cluster distances and patterns of different classes having large inter-cluster distances enable the classification algorithm to work in a proficient way. The problem of this work is overlapping of different classes patterns with feature space 
and can be resolved with the help of two-tier classification method whose aim is to suppress the feature space dimensionality and to enhance the classification efficiency. In two-tier method of classification, initial training of extracted features is performed by the SOM (self-organizing map) neural network followed by the KNN classifier. The advantageous features of two-tier classification include deterministic reproducible results and better final distortion. They collected 65 MRI images and they achieved $94.28 \%$ accuracy.

Singh et al., (2016) proposed method comprising Preprocessing, segmentation and classification. Segmentation is performed by fuzzy c-means clustering algorithm, feature extraction is done by gray level run length matrix (GLRLM) and classification is performed by artificial neural network (ANN). For training and testing phase, they collected 120 real brain MRIs of which 60 abnormal and 60 normal and they achieved $87.50 \%$ accuracy, $75 \%$ of Sensitivity and $100 \%$ of specificity.

Abd-Ellah et al., (2016) proposed CAD system comprising five steps namely MRI preprocessing to eliminate background noise, combination of K-means clustering and Otsu binarization for image segmentation, DWT approach for feature extraction, and PCA method for features dimensionality reduction. MRI classification is performed by kernel support vector machine (KSVM) and they collected 80 MRI images, out of which 70 abnormal and 10 normal images. The $100 \%$ accuracy is achieved by GRB kernel compared to $83.5 \%$ of linear kernel, $72.5 \%$ by multilayer perceptron (MLP) kernel, and $97.5 \%$ by polynomial kernel.

Alfonse et al., (2016) proposed method consist of data acquisition, preprocessing, segmentation is done by employing adaptive thresholding and expectation maximization (EM) algorithm. Fast Fourier Transform (FFT) is employed to extract the feature from MRI data set, Minimal-Redundancy-Maximal-Relevance criterion (MRMR) is used for the selection of features and SVM classifier is employed to classify and to select most valuable features. they collected 100 MR images, among which 20 are normal and 80 images have tumor. The format of image is DICOM with size 512 x 512. They achieved $98.9 \%$ of accuracy.

Saha et al., (2016) proposed rough sets method and impreciseness of set boundaries representation is advantage of rough sets. They collected 100 MRI images from BRATS 2013 and 2015 and they achieved $93.43 \%$ of sensitivity.

Amin et al., (2017) proposed Gaussian filter for noise reduction and improve the image quality. Action and SVM is used for classification of brain tumor. They collected 85 images (39 healthy images and 46 images having tumor) from Nashtar Hospital Multan, 100 images from Harvard dataset in which 65 images are tumor and 35 images are healthy and 126 patient's images from Cancer imaging archive (TCIA) organized RIDER brain image data. They achieved $97.1 \%$ accuracy, $91.9 \%$ sensitivity and $98.0 \%$ specificity.

Bahadure et al., (2017) proposed Berkeley wavelet transformation (BWT) to segment brain tumor and SVM based classifier to improve the accuracy. They collected 22 images of 15 patients from the Digital Imaging and Communications in Medicine (DICOM) dataset and expert radiologists. They achieved $94.2 \%$ specificity, $96.51 \%$ accuracy and $97.72 \%$ sensitivity.

Banerjee et al., (2018) by using multi-sequence MR images they proposed Deep Convolutional Neural Networks (ConvNets) to classify the brain tumors. Three ConvNets proposed by them are trained from scratch, on slices, MRI patches, and multiplanar volumetric slices and they applied this method on existing two ConvNets models for pre-trained VGGNet (16 layers), and ResNet (50 layers) architectures have been applied for transfer learning which are trained on the ImageNet dataset. The ConvNets performance can be evaluated by Leave-one-patient out (LOPO) testing scheme. Multi-planar volumetric dataset is employed to train the model and better accuracy can be achieved by ConvNet. They collected 32 samples of 277 MRI images and utilizing multi-planar MRI slices, they accomplished $97.19 \%$ of accuracy.

Shree et al., (2018) proposed gray-level co-occurrence matrix (GLCM) to extract the features, and to improve performance and complexity reduction, DWT based brain tumor region growing segmentation has been done. To classify brain tumors, the classifier called probabilistic neural network has been utilized. They collected 25 images of DICOM dataset to get 650 samples, out of which, the infected brain tissues are 18 and others are normal. They made use of trained dataset from websites known as www.diacom.com and test dataset. They achieved $100 \%$ accuracy.

Krishna et al., (2018) made the classification and detection of brain tumors into benign and malignant by employing Particle Swarm Optimization (PSO) based Local Linear Radial Basis Function Neural Network (LLRBFNN) model. They collected 200 MRI images of normal and abnormal images for testing and training dataset from the Alzheimer's disease Neuro imaging Initiative (ADNI) public database and Harvard medical school architecture and they achieved $98.7 \%$ accuracy.

Kavin Kumar et al., (2018) proposed a transform called Poisson unbiased risk estimator- linear expansion of 
thresholds (PURE-LET) to denoising an image and for feature extraction, they used combination of Multi-Texton Microstructure Descriptor (MTMD) and Modified Multi-Texton Histogram (MMTH). Feature extraction of the image is done by Gray Level Run Length Matrix (GLRLM) and GLCM, and for classification purpose they used SVM and KNN. The extracted features are used to train Extreme Learning Machine (ELM) and are employed for classification of images. They collected 46 normal and 44 tumor training dataset images and 21 normal and 23 tumor testing images. They achieved accuracy with SVM classifier is $95 \%$, with KNN classifier $80 \%$ and with ELM classifier $91 \%$.

Deepa et al., (2019) proposed method which consists of feature extraction, preprocessing, fusion to accomplish high accuracy in classification, and selection. The average filter is employed to reduce variation in intensity of images in the preprocessing step. The orientation, locality, and frequency are extracted by Gabor wavelet feature extraction which gives texture information to classify. The small subset of features is selected by kernel principal component analysis (KPCA) to enhance the relevancy and to suppress the redundancy of the feature. The GRBF of feature fusion gives the information distinguished from the features having multiple sets. To classify a fused feature adaptive firefly backpropagation neural network is employed. They collected images from the BRATS 2015 dataset comprising 81 MRI images, with 11 normal images, 55 images having malignant tumor and 15 images having benign tumor. They achieved $99.85 \%$ of specificity, $99.84 \%$ of Accuracy and $97.24 \%$ of sensitivity.

Mallick et al., (2019) used deep wavelet autoencoder (DWA) technique to compress image. The deep neural network (DNN) ensures the further classification and has impact on sinking of the feature. The comparison of performance of DWA-DNN classifier has been done with other classifiers such as DNN or autoencoder-DNN, and it has been noticed that the proposed method superior compared to existing methods. Interpretation of medical image dataset is time-consuming process and it is challenging task to handle them. The proposed DWA-DNN classifier gives best result in terms of specificity, accuracy, sensitivity. They collected 19 patients MRI image from RIDER in DICOM format. They achieved $93 \%$ of accuracy.

Song et al., (2019) proposed a noninvasive automatic diagnosis system which is on the basis of machine learning to detect gliomas. Size normalization, standardization, background noise removal, have been done to get standard images. The improvement in the low-contrast standard brain images is done by modified dynamic histogram equalization. Further, pyramid histogram of the oriented gradient, hybrid features, gray-level co-occurrence matrix, intensity-based features and modified completed local binary pattern are extracted from the enhanced images. The particle swarm optimization along with KSVM is adopted to train classifiers. They collected 120 patients original brain MRIs out of which, 81 images are having glioma, 68 images having other kinds of tumors and 57 normal images from shengjing hospital of china medical university and they achieved $98.36 \%$ of accuracy, $99.17 \%$ of sensitivity, and $97.83 \%$ of specificity respectively.

Shakeel P et al., (2019) For classification of brain tumor, (MLBPNN) machine learning based back propagation neural networks system is proposed, which helps pathologists to improve the exactness of threat location and help doctors in studying the picture cell by using bunching calculations and order by recoloring phones qualities. Fractal dimension algorithm is used to extract and then important features are selected by multi fractal detection technique for complexity minimization. The integration of imaging sensor is done through wireless infrared imaging sensor to transfer the data of tumor to clinician to examine the condition of wellbeing and for ultrasound measurements level control, especially for elder patients living in remote zones. Testing and training are two processes in MLBPNN. The classification of tumor images is class I database for area $<500$ and Class II Database for area $>500$. In testing, holes filling is done for the image that has to be tested. This highlights the tumor part by filling the area around tumor helps to find the tumor location exactly Imerode function is used. Accuracy is estimated and tumor area is calculated and thus classified as class I or Class II. They collected $30 \mathrm{MRI}$ images of 21 abnormal and 9 normal from surgical planning laboratory (SPL) dataset and they achieved $93.33 \%$ of accuracy, $71.42 \%$ of sensitivity and $88.88 \%$ of specificity.

Rajesh T et al., (2019) The proposed method includes classification of tumor and extraction of features. For the extraction of features, they used (RST) Rough set theory and for the classification of MRI brain images as abnormal and normal particle swam optimization neural network (PSONN) is tested. They collected 90 images, 60 images are utilized for testing and 30 for training and they achieved $95 \%$ of accuracy as compared to RST-FFNN (Feed-forward neural Network) and RST-FSVM.

Polepaka et al., (2019) The proposed method consists of tumor region identification, preprocessing and tumor region classification. In the preprocessing the filter method is used to reduce the noise and to recognize tumor region location of filtered image they used Bounding Box (BB) algorithm. Exact tumor location is classified by Support Vector Machine (SVM). They collected $101 \mathrm{MRI}$ brain images and they used 50 images of 35 tumor images and 15 without tumor images from openly available dataset from website and they achieved $100 \%$ of segmentation accuracy compared with (EDPSO) 
Enhanced Darwinian Particle Swarm Optimization of accuracy $95 \%$ and Particle Swarm Optimization (PSO) of accuracy $92 \%$.

Deepika et al., (2019) The proposed method consists of Preprocessing, segmentation, dimension reduction, and classification. Extraction of features from preprocessed images is done by local binary pattern (LBP) technique and to convert into grayscale images T2-weighted preprocessing is applied on MR Images. PCA is utilized for feature reduction. In order to classify MR image is abnormal or normal the minimized feature set is directed to SVM classifier. They utilized benchmark dataset Brats15 and Midas: database of human brain which are Healthy. This dataset includes 180 abnormal and 100 normal images, out of $280 \mathrm{MR}$ images. They achieved $100 \%$ of accuracy, sensitivity, and specificity.

Rani et al., (2019) the performance of k-means, fuzzy c-means clustering and KIFCM (integration of k-means and fuzzy c-means) are tested and they proposed ostu thresholding and support vector machine (SVM). They collected 152 images from Brain Web (Simulated Brain database) dataset including MNC extension. 22 brain tumor images from Digital Imaging and Communications in Medicine (DICOM) is second dataset. The third one is 81 images from BRATS dataset. The fourth one is Medinfo and the final dataset is 17 images are gathered from the Harvard Medical School website. They achieved accuracy of $95.45 \%$.

Abiwinanda et al., (2019) proposed convolutional neural network for tumor segmentation and CNN is guided using 3064 T-1 plodding CE-MRI images of brain tumor. They collected images from Jun Cheng previously utilized in his paper. The dataset includes 1426 images with meningioma, 930 images with pituitary tumors and 708 images with glioma. In their guiding phase, they level the images which are utilized to guide the $\mathrm{CNN}$ for each tumors class. Among all available images, they utilized only 700 images, 500 of those images were utilized for guiding phase and other 200 images for validation phase. They achieved $94.68 \%$ of accuracy.

Thillaikkarasi et al., (2019) To classify the tumor efficiently and automatically they proposed novel deep learning algorithm (kernel based CNN) including M-SVM. This work includes some steps they are preprocessing, feature extraction, image classification and brain tumor segmentation. By Laplacian of Gaussian filtering method (LoG) along with Contrast Limited Adaptive Histrogram Equalization (CLAHE) the MRI image is improved and on the basis of tumor shape, position and surface features, brain features can be obtained. Consequently, based on the selected features image classification is performed by utilizing
M-SVM. They collected 40 MRI images of 25 patients and are classified into two types abnormal and normal. They achieved $84 \%$ of segmentation accuracy.

Sharma et al., (2019) proposed Differential Evolution algorithm along with OTSU method and collected 56 MRI images of 56 patients consisting 18 patients who are healthy and 38 brain tumor patients and gained $94.73 \%$ of accuracy.

Thah et al., (2019) proposed Enhanced Convolutional Neural Networks (ECNN) with accession of loss function by BAT algorithm for Skull stripping and for spontaneous classification of brain tumor and image improvement algorithms are utilized for pre-processing. They collected dataset from BRATS 2015 and they concluded that current CNN method gives only $89 \%$ accuracy whereas the proposed ECNN model gives higher accuracy of $92 \%$ respectively.

Chander et al., (2020) They proposed that MRI image can be divided into multiple segments by adaptive k-means clustering and by using Support Vector Machine classifier segmented images are classified. They collected forty MR images of malignant and Benign tumors from Harvard University medical Image Repository. They achieved 93\% of accuracy using linear kernel method and segmentation accuracy is $99.7 \%$.

Chaudhary et al., (2020) They proposed K-Means for segmentation and DWT is used to extract features. For the classification between malignant and benign tumor SVM is applied at last. They used 6 images for testing their code from Rajendra institute of medical science and they achieved $94.6 \%$ of accuracy.

Vijh et al., (2020) To find the optimal threshold value, adaptive particle swarm optimization including OTSU is proposed. In order to eliminate the noise and enhance the image quality Anisotropic diffusion filtering is employed on brain MRI. For performing classification and guiding the convolutional neural network, data is provided by extracted features. They collected $40 \mathrm{MR}$-free non-tumored images and 61 IBSR (Internet Brain Segmentation Repository) tumored images of magnetic resonance and gained $98 \%$ of accuracy.

Ansari M et al., (2020) proposed median filtering to denoise the image and Morphological Operation for Image Segmentation. The DWT and GLCM is utilized for feature extraction and SVM are utilized for segmentation of brain tumor as benign and malignant. They used 5 MRI images for testing their code these images are JPEG/JPG format and they achieved $98.91 \%$ of accuracy.

Gokulalakshmi et al., (2020) proposed SVM classifier and K-means clustering for classification. For feature extraction 
Grey-Level Co-occurrence Matrix (GLCM) and Discrete Wavelet Transformation (DWT) are used. They collected 750 samples of 30 images from DICOM dataset and They achieved $94 \%$ of accuracy.

Chander $\mathbf{P}$ et al., (2020) proposed adaptive K-Means clustering algorithm for segmentation and SVM classifier is used for classification. Discrete Wavelet Transformation (DWT) and Grey-Level Co-occurrence Matrix (GLCM) are utilized for feature extraction. Forty MR images of malignant and Benign tumor are collected from Harvard University medical Image Repository. They achieved $99.7 \%$ of segmentation accuracy and $93 \%$ of Classification accuracy.

\section{A. Review table}

Table 1. Review table of classification methodologies.

\begin{tabular}{|c|c|c|c|c|c|}
\hline $\begin{array}{l}\text { Sl. } \\
\text { N } \\
\text { o }\end{array}$ & $\begin{array}{l}\text { Auth } \\
\text { ors }\end{array}$ & Year & Methods & Dataset & Accuracy \\
\hline 1 & $\begin{array}{l}\text { Arak } \\
\text { eri }\end{array}$ & 2013 & $\begin{array}{l}\mathrm{SVM}+\mathrm{A} \\
\mathrm{NN}+\mathrm{KN} \\
\mathrm{N}\end{array}$ & $\begin{array}{l}550 \text { patients } \\
\text { MRI images } \\
\text { from shirdi } \\
\text { sai cancer } \\
\text { hospital, } \\
\text { manipal, } \\
\text { India }\end{array}$ & $\begin{array}{l}\text { SVM(96.4 } \\
2 \%) \mathrm{ANN}( \\
94.18 \%) \mathrm{K} \\
\mathrm{NN}(89.09 \\
\%)\end{array}$ \\
\hline 2 & $\begin{array}{l}\text { Amie } \\
\mathrm{n}\end{array}$ & 2013 & $\begin{array}{l}\mathrm{BPNN}+\mathrm{P} \\
\mathrm{CA}\end{array}$ & $\begin{array}{l}58 \\
\text { MRIimages } \\
\text { of } 3 \mathrm{MRI} \\
\text { human brain } \\
\text { dataset }\end{array}$ & $96.8 \%$ \\
\hline 3 & $\begin{array}{l}\text { Jayac } \\
\text { hand } \\
\text { ran }\end{array}$ & 2013 & $\begin{array}{l}\text { Anisotro } \\
\text { pic } \\
\text { filter+ } \\
\text { FSVM }\end{array}$ & $\begin{array}{l}80 \text { brain } \\
\text { MRI images }\end{array}$ & $95.80 \%$ \\
\hline 4 & $\begin{array}{l}\text { Sund } \\
\text { arara } \\
\mathrm{j}\end{array}$ & 2014 & $\begin{array}{l}\text { Gaussian } \\
\text { filter }+ \\
\mathrm{PCA}+\mathrm{K} \\
\mathrm{NN} \\
+ \text { Linear } \\
\text { SVM }\end{array}$ & $\begin{array}{l}50 \text { CT brain } \\
\text { images of } \\
\text { which } 10 \text { are } \\
\text { of normal } \\
\text { brain images } \\
\text { and } 40 \text { are of } \\
\text { abnormal } \\
\text { brain images } \\
\text { from } \\
\text { Department } \\
\text { of } \\
\text { Radiology, } \\
\text { Rajah } \\
\text { Muthiah } \\
\text { Medical } \\
\text { College } \\
\text { Hospital }\end{array}$ & $\begin{array}{l}\text { KNN(92 } \\
\%), \text { Linear } \\
\text { SVM } \\
(94 \%)\end{array}$ \\
\hline
\end{tabular}

\begin{tabular}{|c|c|c|c|c|c|}
\hline & & & & $(\mathrm{RMMCH})$ & \\
\hline 5 & $\begin{array}{l}\text { Natte } \\
\text { shan, } \\
\mathrm{N}\end{array}$ & 2015 & $\begin{array}{l}\text { Wiener } \\
\text { filter+CL } \\
\text { AHE+ } \\
\text { SVM }\end{array}$ & $\begin{array}{l}83 \text { tumor } \\
\text { and } 9 \text { non } \\
\text { tumor gray } \\
\text { scale images } \\
\text { from } \\
\text { DICOM }\end{array}$ & $\begin{array}{l}\text { Quadratic } \\
\text { kernel } \\
\text { function } \\
(85.40 \%) \text {, } \\
\operatorname{RBF}(83.6 \\
1 \%)\end{array}$ \\
\hline 6 & $\begin{array}{l}\text { Anit } \\
\text { ha, V }\end{array}$ & 2016 & $\begin{array}{l}\text { Adaptive } \\
\text { piller K- } \\
\text { means } \\
\text { algorith } \\
\mathrm{m}+\mathrm{SOM} \\
+\mathrm{KNN}\end{array}$ & $\begin{array}{l}65 \mathrm{MRI} \\
\text { images }\end{array}$ & $94.28 \%$ \\
\hline 7 & $\begin{array}{l}\text { Sing } \\
\mathrm{h}\end{array}$ & 2016 & $\begin{array}{l}\text { FCM+G } \\
\text { LRLM+ } \\
\text { ANN }\end{array}$ & $\begin{array}{l}120 \mathrm{MRI} \\
\text { images 0f } 60 \\
\text { are normal } \\
\text { and } 60 \\
\text { abnormal } \\
\text { images }\end{array}$ & $87.50 \%$ \\
\hline 8 & $\begin{array}{l}\text { Abd- } \\
\text { Ellah }\end{array}$ & 2016 & $\begin{array}{l}\text { DWT+P } \\
\mathrm{CA}+ \\
\mathrm{KSVM}\end{array}$ & $\begin{array}{l}80 \text { MRI } \\
\text { images of } 10 \\
\text { are normal } \\
\text { and } 70 \text { are } \\
\text { abnormal }\end{array}$ & $\begin{array}{l}\text { GRB }(100 \\
\%) \text {, linear } \\
\text { kernal } \\
(72.50 \%), \\
\text { MLP(97.5 } \\
\%) \text { and } \\
\text { polynomia } \\
1 \\
\text { kernal(97. } \\
5 \%)\end{array}$ \\
\hline 9 & $\begin{array}{l}\text { Alfo } \\
\text { nse }\end{array}$ & 2016 & $\begin{array}{l}\text { EM+FFT } \\
+\mathrm{MRMR} \\
+\mathrm{SVM}\end{array}$ & $\begin{array}{l}100 \mathrm{MRI} \\
\text { images of } 20 \\
\text { are normal } \\
\text { and } 80 \text { are } \\
\text { abnormal }\end{array}$ & $98.9 \%$ \\
\hline 10 & Saha & 2016 & $\begin{array}{l}\text { Rough } \\
\text { sets }\end{array}$ & $\begin{array}{l}100 \mathrm{MRI} \\
\text { images from } \\
\text { BRATS } \\
2013 \text { to } \\
2015\end{array}$ & $\begin{array}{l}93.43 \% \\
\text { sensitivity }\end{array}$ \\
\hline 11 & $\begin{array}{l}\text { Gari } \\
\text { ma }\end{array}$ & 2016 & $\begin{array}{l}\text { Naive } \\
\text { Bayes+S } \\
\text { VM }\end{array}$ & $\begin{array}{l}110 \text { brain } \\
\text { MRI images } \\
\text { is from } \\
\text { Yatharth } \\
\text { Hospital, } \\
\text { Noida }\end{array}$ & $\begin{array}{l}\text { Naive } \\
\text { Bayes } \\
(87.23), \\
\text { SVM(91.4 } \\
9 \%) \text { of } \\
\text { efficiency }\end{array}$ \\
\hline 12 & $\begin{array}{l}\text { Ami } \\
n\end{array}$ & 2017 & $\begin{array}{l}\text { Gaussian } \\
\text { filter+SV } \\
\mathrm{M}\end{array}$ & $\begin{array}{l}85 \text { images } \\
\text { of } 46 \text { are } \\
\text { tumor and } \\
39 \text { are non } \\
\text { tumor } \\
\text { images from } \\
\text { Nashtar } \\
\text { Hospital } \\
\text { Multan and }\end{array}$ & $97.1 \%$ \\
\hline
\end{tabular}




\begin{tabular}{|c|c|c|c|c|c|c|c|c|c|c|c|}
\hline & & & & $\begin{array}{l}100 \text { images } \\
\text { of } 65 \text { are } \\
\text { tumor and } \\
35 \text { are non } \\
\text { tumor } \\
\text { images from } \\
\text { Harvard }\end{array}$ & & & $\begin{array}{l}\text { n } \\
\text { Kum } \\
\text { ar }\end{array}$ & & $\begin{array}{l}\text { ET+MM } \\
\text { TH+MT } \\
\text { MDGLC } \\
\text { M+GLR } \\
\text { LM+SV } \\
\text { M+KNN } \\
+ \text { ELM }\end{array}$ & $\begin{array}{l}\text { and } 44 \\
\text { abnormal } \\
\text { MRI images }\end{array}$ & $\begin{array}{l}\%), \\
\text { KJNN(80 } \\
\%), \\
\text { ELM(91\% } \\
)\end{array}$ \\
\hline & & & & $\begin{array}{l}\text { dataset of } \\
126 \text { patient's } \\
\text { images from } \\
\text { Cancer } \\
\text { imaging } \\
\text { archive } \\
\text { (TCIA)orga } \\
\text { nized } \\
\text { RIDER }\end{array}$ & & 18 & $\begin{array}{l}\text { Deep } \\
a, A\end{array}$ & 2019 & $\begin{array}{l}\text { Average } \\
\text { filter+Ga } \\
\text { bor } \\
\text { wavelet+ } \\
\text { KPCA+ } \\
\text { GRBF }\end{array}$ & $\begin{array}{l}81 \text { MRI } \\
\text { images of } 55 \\
\text { are } \\
\text { malignant } \\
\text { tumor, } 15 \\
\text { benign } \\
\text { tumor and } \\
11 \text { normal } \\
\text { images }\end{array}$ & $99.84 \%$ \\
\hline & & & & $\begin{array}{l}\text { brain image } \\
\text { data. }\end{array}$ & & 19 & $\begin{array}{l}\text { Malli } \\
\text { ck }\end{array}$ & 2019 & $\begin{array}{l}\mathrm{DWA}+\mathrm{D} \\
\mathrm{NN}\end{array}$ & $\begin{array}{l}19 \text { patients } \\
\text { MRI images }\end{array}$ & $93 \%$ \\
\hline 13 & Baha & 2017 & $\mathrm{BWT}+\mathrm{S}$ & $22 \mathrm{MRI}$ & $96.51 \%$ & & & & & from RIDER & \\
\hline & dure & & VM & $\begin{array}{l}\text { images of } 15 \\
\text { patients } \\
\text { from } \\
\text { DICOM } \\
\text { dataset and } \\
\text { expert } \\
\text { radiologist }\end{array}$ & & 20 & Song & 2019 & $\begin{array}{l}\text { GLCM+ } \\
\text { PSO+KS } \\
\text { VM }\end{array}$ & $\begin{array}{l}120 \text { patients } \\
\text { original } \\
\text { MRI images } \\
\text { of } 181 \\
\text { images with } \\
\text { glioma,57 } \\
\text { images with }\end{array}$ & $98.36 \%$ \\
\hline 14 & $\begin{array}{l}\text { Shre } \\
\text { e }\end{array}$ & 2018 & $\begin{array}{l}\mathrm{DWT}+\mathrm{G} \\
\mathrm{LCM}+\mathrm{P} \\
\mathrm{NN}\end{array}$ & $\begin{array}{l}650 \text { samples } \\
\text { from } 25 \\
\text { MRI images } \\
\text { of } 7 \text { are } \\
\text { normal and } \\
18 \text { are } \\
\text { abnormal } \\
\text { from } \\
\text { DICOM } \\
\text { dataset }\end{array}$ & $95 \%$ & & & & & $\begin{array}{l}\text { normal and } \\
68 \text { images } \\
\text { with other } \\
\text { kinds of } \\
\text { tumors from } \\
\text { shengjing } \\
\text { hospital of } \\
\text { china } \\
\text { medical } \\
\text { university }\end{array}$ & \\
\hline 15 & $\begin{array}{l}\text { Bane } \\
\text { rjee }\end{array}$ & 2018 & $\begin{array}{l}\text { ConvNet } \\
\text { s }\end{array}$ & $\begin{array}{l}32 \text { samples } \\
\text { from } 277 \\
\text { MRI images }\end{array}$ & $97.19 \%$ & 21 & $\begin{array}{l}\text { Shak } \\
\text { eel, P }\end{array}$ & 2019 & $\begin{array}{l}\text { MLBPN } \\
\mathrm{N}\end{array}$ & $\begin{array}{l}30 \text { MRI } \\
\text { images of } 21 \\
\text { are }\end{array}$ & $93.33 \%$ \\
\hline 16 & $\begin{array}{l}\text { Krish } \\
\text { na }\end{array}$ & 2018 & $\begin{array}{l}\text { PSO+LL } \\
\text { RBFNN }\end{array}$ & $\begin{array}{l}200 \text { MRI } \\
\text { images from } \\
\text { the Harvard } \\
\text { medical } \\
\text { school }\end{array}$ & $98.7 \%$ & & & & & $\begin{array}{l}\text { abnormal } \\
\text { and } 9 \\
\text { normal } \\
\text { images from } \\
\text { SPL dataset }\end{array}$ & \\
\hline & & & & $\begin{array}{l}\text { architecture } \\
\text { and } \\
\text { Alzheimer's } \\
\text { disease } \\
\text { Neuroimagi } \\
\text { ng Initiative }\end{array}$ & & 22 & $\begin{array}{l}\text { Rajes } \\
\mathrm{h}\end{array}$ & 2019 & $\begin{array}{l}\text { RST+PS } \\
\text { ONN }\end{array}$ & $\begin{array}{l}90 \text { MRI } \\
\text { images are } \\
\text { used for } \\
\text { training and } \\
60 \text { are used } \\
\text { for testing }\end{array}$ & $95 \%$ \\
\hline & & & & $\begin{array}{l}\text { (ADNI) } \\
\text { public } \\
\text { databaset }\end{array}$ & & 23 & $\begin{array}{l}\text { Pole } \\
\text { paka }\end{array}$ & 2019 & $\begin{array}{l}\mathrm{BB}+\mathrm{SV} \\
\mathrm{M}\end{array}$ & $\begin{array}{l}50 \text { MRI } \\
\text { images of } 35 \\
\text { tumor and }\end{array}$ & $100 \%$ \\
\hline 17 & Kavi & 2018 & PURE-L & 46 normal & SVM(95 & & & & & 15 non & \\
\hline
\end{tabular}




\begin{tabular}{|c|c|c|c|c|c|}
\hline & & & & $\begin{array}{l}\text { tumor } \\
\text { imagesfrom } \\
\text { publicly } \\
\text { available } \\
\text { dataset from } \\
\text { world wide } \\
\text { web }\end{array}$ & \\
\hline 24 & $\begin{array}{l}\text { Deep } \\
\text { ika }\end{array}$ & 2019 & $\begin{array}{l}\mathrm{LBP}+\mathrm{PC} \\
\mathrm{A}+\mathrm{SVM}\end{array}$ & $\begin{array}{l}280 \text { MRI } \\
\text { images of } \\
180 \\
\text { abnormal } \\
\text { and } 100 \\
\text { normal } \\
\text { images from } \\
\text { MIDAS and } \\
\text { BRATS201 } \\
5\end{array}$ & $100 \%$ \\
\hline 25 & Rani & 2019 & $\begin{array}{l}\text { SVM+KI } \\
\text { FCM }\end{array}$ & $\begin{array}{l}152 \text { MRI } \\
\text { images from } \\
\text { brain web } \\
\text { dataset ,22 } \\
\text { MRI images } \\
\text { from } \\
\text { DICOM and } \\
81 \text { MRI } \\
\text { images from } \\
\text { BRATS } \\
\text { dataset }\end{array}$ & $95.45 \%$ \\
\hline 26 & $\begin{array}{l}\text { Abiw } \\
\text { inand } \\
\text { a }\end{array}$ & 2019 & $\mathrm{CNN}$ & $\begin{array}{l}708 \text { MRI } \\
\text { images with } \\
\text { glioma, } \\
1426 \text { MRI } \\
\text { images with } \\
\text { meningioma } \\
\text {, } 930 \text { MRI } \\
\text { images with }\end{array}$ & \\
\hline 27 & $\begin{array}{l}\text { Thill } \\
\text { aikka } \\
\text { rasi }\end{array}$ & 2019 & $\begin{array}{l}\text { Kenel } \\
\text { based } \\
\text { CNN+C } \\
\text { LAHE+ } \\
\text { MSVM }\end{array}$ & $\begin{array}{l}40 \text { MRI } \\
\text { images of } 25 \\
\text { patients }\end{array}$ & $84 \%$ \\
\hline 28 & $\begin{array}{l}\text { Shar } \\
\text { ma }\end{array}$ & 2019 & $\begin{array}{l}\text { Dfferenti } \\
\text { al } \\
\text { evolution } \\
\text { algorith } \\
\text { m+otsu } \\
\text { method }\end{array}$ & $\begin{array}{l}56 \mathrm{MRI} \\
\text { images of } 56 \\
\text { patients } \\
\text { including } 18 \\
\text { non tumor } \\
\text { and } 38 \\
\text { tumors }\end{array}$ & $94.73 \%$ \\
\hline 29 & $\begin{array}{l}\text { Thah } \\
\text { a }\end{array}$ & 2019 & ECNN & $\begin{array}{l}\text { BRATS } \\
2015\end{array}$ & $92 \%$ \\
\hline 30 & $\begin{array}{l}\text { Chan } \\
\text { der }\end{array}$ & 2020 & $\begin{array}{l}\text { Adaptive } \\
\mathrm{K} \text { means } \\
\text { clusterin } \\
\text { g+svm }\end{array}$ & $\begin{array}{l}40 \text { MRI } \\
\text { images of } \\
\text { Benign and } \\
\text { malignant }\end{array}$ & $99.7 \%$ \\
\hline
\end{tabular}

\begin{tabular}{|c|c|c|c|c|c|}
\hline & & & & $\begin{array}{l}\text { tumors from } \\
\text { Harvard } \\
\text { University } \\
\text { medical } \\
\text { Image } \\
\text { Repository }\end{array}$ & \\
\hline 31 & $\begin{array}{l}\text { Chau } \\
\text { dhary }\end{array}$ & 2020 & $\begin{array}{l}\mathrm{K} \\
\text { means+D } \\
\mathrm{WT}+\mathrm{SV} \\
\mathrm{M}\end{array}$ & $\begin{array}{l}6 \mathrm{MRI} \\
\text { imagesfor } \\
\text { testing from } \\
\text { Rajendra } \\
\text { institute of } \\
\text { medical } \\
\text { science }\end{array}$ & $94.6 \%$ \\
\hline 32 & Vijh & 2020 & $\begin{array}{l}\text { Adaptive } \\
\text { PSO+OT } \\
\text { SU }\end{array}$ & $\begin{array}{l}61 \text { tumor } \\
\text { and } 40 \text { non } \\
\text { tumor MRI } \\
\text { images from } \\
\text { IBSR }\end{array}$ & $98 \%$ \\
\hline 33 & $\begin{array}{l}\text { Ansa } \\
\text { ri }\end{array}$ & 2020 & $\begin{array}{l}\text { Morphol } \\
\text { ogocal } \\
\text { operation } \\
\text { +DWT+ } \\
\text { GLCM+ } \\
\text { SVM }\end{array}$ & $\begin{array}{l}5 \\
\text { MRIimages } \\
\text { for testing } \\
\text { their code }\end{array}$ & $98.91 \%$ \\
\hline 34 & $\begin{array}{l}\text { Goku } \\
\text { lalak } \\
\text { shmi }\end{array}$ & 2020 & $\begin{array}{l}\mathrm{K} \\
\text { means+D } \\
\mathrm{WT}+\mathrm{GL} \\
\mathrm{CM}+\mathrm{SV} \\
\mathrm{M}\end{array}$ & $\begin{array}{l}750 \text { samples } \\
\text { of } 30 \mathrm{MRI} \\
\text { images from } \\
\text { DICOM } \\
\text { dataset }\end{array}$ & $94 \%$ \\
\hline 35 & $\begin{array}{l}\text { Chan } \\
\text { der }\end{array}$ & 2020 & $\begin{array}{l}\text { K } \\
\text { means+D } \\
\text { WT+ } \\
\text { GLCM+ } \\
\text { SVM }\end{array}$ & $\begin{array}{l}40 \text { MRI } \\
\text { tumor and } \\
\text { non-tumor } \\
\text { images from } \\
\text { Harvard } \\
\text { University } \\
\text { medical } \\
\text { Image } \\
\text { Repository }\end{array}$ & $93 \%$ \\
\hline
\end{tabular}

\section{III.CONCLUSION}

One of the leading diseases in the world is brain tumors. So, it is very important to detect and classify brain tumor at early stage. MRI brain images are widely utilized to classify brain tumors. Categorization of brain tumor from MRI is classified into four phases. Median filter is included in first phase preprocessing to eliminate the noise. Brain tumor segmentation using otsu binarization and K-means clustering is included in the second phase. Transform feature extraction utilizing DWT and spatial feature extraction utilizing GLCM are included in third phase. DWT is widely used to decompose the image and improve the 
resolution by removing the unwanted region. PCA is utilized to minimize the features to maintain the classification accuracy of brain MR images. The use of SVM along with the appropriate kernel techniques can help in achieving high accuracy.

\section{REFERENCES}

[1] Arakeri, Megha P.; G. Ram Mohana Reddy. Computer-aided diagnosis system for tissue characterization of brain tumor on magnetic resonance images. Signal, Image and Video Processing, 2013, 9, PP.409-425.

[2] Amien; Magdi BM; Ahmed Abd-elrehman; Walla Ibrahim. An intelligent-model for automatic brain-tumor diagnosis based-on MRI images. International Journal of Computer Applications 2013, 72, PP.21-24.

[3] Jayachandran, A., and R. Dhanasekaran. "Brain tumor detection and classification of MR images using texture features and fuzzy SVM classifier." Research Journal of Applied Sciences, Engineering and Technology 2013, 12, PP.2264-2269.

[4] Sundararaj, G. Kharmega, and V. Balamurugan. "Robust classification of primary brain tumor in Computer Tomography images using K-NN and linear SVM." In 2014 International Conference on Contemporary Computing and Informatics (IC3I), 2014, PP.1315-1319.

[5] Natteshan, N. V. S., and J. Angel Arul Jothi. "Automatic classification of brain MRI images using SVM and neural network classifiers." In Advances in intelligent informatics, Springer, Cham 2015, PP.19-30.

[6] Anitha, V., and S. Murugavalli. "Brain tumour classification using two-tier classifier with adaptive segmentation technique." IET computer vision, 2016, 10, PP.9-17.

[7] Singh, Amritpal. "Detection of brain tumor in MRI images, using fuzzy C-means segmented images and artificial neural network." In Proceedings of the International Conference on Recent Cognizance in Wireless Communication \& Image Processing, Springer, New Delhi.

2016, PP.123-131.

[8] Abd-Ellah, Mahmoud Khaled, Ali Ismail Awad, Ashraf AM Khalaf, and Hesham FA Hamed. "Classification of brain tumor MRIs using a kernel support vector machine." In International Conference on Well-Being in the Information Society, Springer, Cham, 2016, PP.151-160.

[9] Alfonse, Marco, and Abdel-Badeeh M. Salem. "An automatic classification of brain tumors through MRI using support vector machine." Egyptian Computer Science Journal (ISSN: 1110 - 2586) ,2016, PP.40.

[10] Saha, Rupsa, Ashish Phophalia, and Suman K. Mitra. "Brain tumor segmentation from multimodal mr images using rough sets." In International Conference on Computer Vision, Graphics, and Image processing, Springer, Cham, 2016,. PP.133-144.

[11] Singh, Garima, and M. A. Ansari. "Efficient detection of brain tumor from MRIs using K-means segmentation and normalized histogram." In 2016 1st India International Conference on Information Processing (IICIP), 2016, PP.1-6.

[12] Amin, Javeria, Muhammad Sharif, Mussarat Yasmin, and Steven Lawrence Fernandes. "A distinctive approach in brain tumor detection and classification using MRI." Pattern Recognition Letters, Elsevier , 2017, PP.1-10 .

[13] Bahadure, Nilesh Bhaskarrao, Arun Kumar Ray, and Har Pal Thethi. "Image analysis for MRI based brain tumor detection and feature extraction using biologically inspired BWT and SVM." International journal of biomedical imaging, hindawi, 2017, PP.1-12.
[14] Banerjee, Subhashis, Sushmita Mitra, Francesco Masulli, and Stefano Rovetta. "Brain Tumor Detection and Classification from Multi-sequence MRI: Study Using ConvNets." In International MICCAI Brainlesion Workshop, Springer, 2018, PP.170-179.

[15] Shree, N. Varuna, and T. N. R. Kumar. "Identification and classification of brain tumor MRI images with feature extraction using DWT and probabilistic neural network." Brain informatics 5, Springer, 2018,1, PP. 23-30

[16] Krishna, T. Gopi, K. V. N. Sunitha, and S. Mishra. "Detection and classification of brain tumor from MRI medical image using wavelet transform and PSO based LLRBFNN algorithm." International Journal of Computer Sciences and Engineering 6, 2018, 1, PP.18-23.

[17] Kavin Kumar, K., Meera Devi, and S. Maheswaran. "An Efficient Method for Brain Tumor Detection Using Texture Features and SVM Classifier in MR Images." Asian Pacific Journal of Cancer Prevention: APJCP 19, no. 10, PP. 2789-2794, 2018.

[18] Deepa, A. R., and WR Sam Emmanuel. "An efficient detection of brain tumor using fused feature adaptive firefly backpropagation neural network." Multimedia Tools and Applications 78, Springer, 2019, 9 , PP.11799-11814.

[19] Mallick, Pradeep Kumar, Seuc Ho Ryu, Sandeep Kumar Satapathy, Shruti Mishra, Gia Nhu Nguyen, and Prayag Tiwari. "Brain MRI image classification for cancer detection using deep wavelet autoencoder-based deep neural network." IEEE Access 7, PP.46278-46287, 2019 IEEE.

[20] Song, Guoli, Zheng Huang, Yiwen Zhao, Xingang Zhao, Yunhui Liu, Min Bao, Jianda Han, and Peng Li. "A noninvasive system for the automatic detection of gliomas based on hybrid features and PSO-KSVM." IEEE Access 7, 2019, PP.13842-13855.

[21] Shakeel, P. Mohamed, Tarek E. El Tobely, Haytham Al-Feel, Gunasekaran Manogaran, and S. Baskar. "Neural network based brain tumor detection using wireless infrared imaging sensor." IEEE Access 7, 2019, PP.5577-5588.

[22] Rajesh, T., R. Suja Mani Malar, and M. R. Geetha. "Brain tumor detection using optimisation classification based on rough set theory." Cluster Computing, Springer. 2019, 22, no 6, PP.13853-13859.

[23] Polepaka, Sanjeeva, Ch Srinivasa Rao, and M. Chandra Mohan. "A Brain Tumor: Localization Using Bounding Box and Classification Using SVM." Innovations in Electronics and Communication Engineering, Springer, Singapore ,2019, 33, PP. 61-70.

[24] Deepika, Kancherla, Jyostna Devi Bodapati, and Ravu Krishna Srihitha. "An Efficient Automatic Brain Tumor Classification Using LBP Features and SVM-Based Classifier." In Proceedings of International Conference on Computational Intelligence and Data Engineering, 2019, PP.163-170, 2019 Springer, Singapor.

[25] Rani, Rajneesh, and Aman Kamboj. "Brain Tumor Classification for MR Imaging Using Support Vector Machine." In Progress in Advanced Computing and Intelligent Engineering, Springer, Singapore,2019, PP.165-176.

[26] Abiwinanda, Nyoman, Muhammad Hanif, S. Tafwida Hesaputra, Astri Handayani, and Tati Rajab Mengko. "Brain tumor classification using convolutional neural network." In World Congress on Medical Physics and Biomedical Engineering 2018, , Springer, Singapore, 2019, PP.183-189.

[27] Thillaikkarasi, R., and S. Saravanan. "An enhancement of deep learning algorithm for brain tumor segmentation using kernel based CNN with M-SVM." Journal of medical systems, springer , 2019, PP.43, 84. 
[28] Sharma, Anshika, Sushil Kumar, and Shailendra Narayan Singh. "Brain tumor segmentation using DE embedded OTSU method and neural network." Multidimensional Systems and Signal Processing 30, springer , 2019,3 ,1263-1291.

[29] Thaha, M. Mohammed, K. Pradeep Mohan Kumar, B. S. Murugan, S. Dhanasekeran, P. Vijayakarthick, and A. Senthil Selvi. "Brain tumor segmentation using convolutional neural networks in MRI images." Journal of medical systems 43, no. 9 , p p 43:294 2019 springer.

[30] Chander, P. Sharath, J. Soundarya, and R. Priyadharsini. "Brain Tumour Detection and Classification Using K-Means Clustering and SVM Classifier." In RITA 2018, Springer, Singapore, 2020, PP.49-63.

[31] Chaudhary, Atish, and Vandana Bhattacharjee. "An efficient method for brain tumor detection and categorization using MRI images by K-means clustering \& DWT." International Journal of Information Technology 12, springer, 2020, 1, PP.141-148.

[32] Vijh, Surbhi, Shilpi Sharma, and Prashant Gaurav. "Brain Tumor Segmentation Using OTSU Embedded Adaptive Particle Swarm Optimization Method and Convolutional Neural Network." In Data Visualization and Knowledge Engineering, Springer, 2020, PP.171-194.

[33] Ansari, M. A., Rajat Mehrotra, and Rajeev Agrawal. "Detection and classification of brain tumor in MRI images using wavelet transform and support vector machine." Journal of Interdisciplinary Mathematics, ISSN: 0972-0502, 2020, PP.1-12.

[34] Gokulalakshmi, A., S. Karthik, N. Karthikeyan, and M. S. Kavitha. "ICM-BTD: improved classification model for brain tumor diagnosis using discrete wavelet transform-based feature extraction and SVM classifier." Soft Computing, Springer ,2020, PP.1-11.

[35] Chander, P. Sharath, J. Soundarya, and R. Priyadharsini. "Brain Tumour Detection and Classification Using K-Means Clustering and SVM Classifier." In RITA 2018, Springer, Singapore ,2020, PP.49-63.

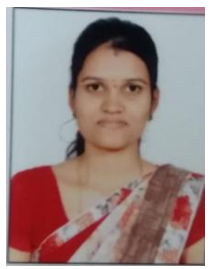

Sangeeta Working as assistant professor at Godutai engineering college for women, Klaburagi.She completed her M.Tech in Poojya Doddappa Appa College of Engineering, Kalabuaragi, Karnataka. She is pursuing part time Ph.D.in Poojya Doddappa Appa College of Engineering, Kalabuaragi, Karnataka, India.Her research area is Biomedical image processing.

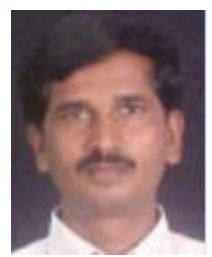

Dr. Nagendra $\mathbf{H}$ received his Ph.D. in Biomedical Signal Processing (Electrical Engineering Department) from Indian Institute of Technology Roorkee, Roorkee, India, 2016 and M.Tech. In Power Electronic (PDA Engg. College,Kalaburagi) from Vishwesharaya Technological University Belagavi, karnataka, India, 2002.B.E. in Electronics and Communication Engineering (PDA Engg. College, kalabuaragi) from Gulbarga University, kalaburagi, Karnataka, India, 1993. he has published many research papers in National and International Journals / Conferences and also presented her research findings in various National and International conferences. Working as Associate Professor in Electronics and Communication, Poojya Doddappa Appa College of Engineering, Kalabuaragi, Karnataka, India from May 2005 to present. He is guiding four students for their $\mathrm{Ph}$. D degree under VTU in biomedical signal and image processing and evaluation of cognitive behaviour under various interventions such as yoga, video games, physical execises etc 